\title{
Estudio Teórico de la Reactividad Química y Biológica de Cisplatino y algunos Derivados con Actividad Anticancerosa
}

\author{
Jesús M. López, Adolfo E. Ensuncho y Juana Robles \\ Universidad de Córdoba, Departamento de Química, Grupo de Química Computacional, \\ Cra. 6 № 74-103, Córdoba-Colombia (e-mail: jmanuellopez.gqc@gmail.com, adem9abril@hotmail.com \\ juanarobles2003@yahoo.com)
}

Recibido Nov. 13, 2012; Aceptado Dic. 17, 2012; Versión final recibida Feb. 17, 2013

\begin{abstract}
Resumen
Se investigó la estructura y reactividad química de algunos derivados del fármaco anticanceroso cisplatino coordinados a ligandos bioactivos al nivel mPW1PW/LANL2DZ en el marco conceptual de la teoría del funcional de la densidad. El conjunto de descriptores globales de la reactividad permitió analizar la naturaleza reactiva de estos complejos. La estabilidad de los complejos aumentó en la fase acuosa. Las geometrías predichas para cisplatino, carboplatino y cis-dicloro[L-N,O-Ornitina]platino(II) mostraron concordancia con los datos de difracción de rayos X experimentales. Asimismo, las geometrías optimizadas en fase acuosa mostraron mejores resultados que las predichas en fase gas. El índice de electrofilicidad global calculado para los complejos cis-dicloro[(L,D)-N,O-Ornitina]platino(II), fue mayor que el predicho para cisplatino, implicando que la unión de estos ligandos favorece la reactividad de estos complejos.
\end{abstract}

Palabras clave: cisplatino, reactividad química, cálculos DFT, índice de electrofilicidad

\section{Theoretical Study of Chemistry Reactivity and Biological of Cisplatin and some Derivatives with Anticancer Activity}

\begin{abstract}
The structure and chemical reactivity of some derivatives of the anticancer drug cisplatin coordinated with bioactive ligands was investigated at mPW1PW/LANL2DZ level theory in the conceptual density functional theory. Global descriptors allowed analyzing the reactive nature of these complexes. The stability of the complexes increases in aqueous phase. Calculated geometries of the complexes cisplatin, carboplatín and cis-dichloro[(L,D)-Ornithine]platinum(II) are in agreement with their available X-ray data. The electrophilicity index calculated for the complexes were higher than those predicted for cisplatin, implying that the binding of these ligands favors the reactivity of these complexes
\end{abstract}

Keywords: cisplatin, chemical reactivity, DFT calculations, electrophilicity index 


\section{INTRODUCCIÓN}

El desarrollo de agentes anticancerosos basados en metales se inició con el complejo de coordinación cisdiclorodiaminoplatino(II), clínicamente conocido como cisplatino (Abramkin et al., 2010; Jungwirth et al., 2012; Beck y Brubaker, 1973). Su actividad citostática fue descubierta, como hecho fortuito, por el biofísico Barnett Rosenberg a finales de los sesentas (Rosenberg et al., 1969), mientras estudiaba los efectos de los campos eléctricos sobre crecimiento de cultivos de células de Escherichia coli. Inicialmente, se planteó como hipótesis que la división celular se detendría por efecto de los campos eléctricos locales. No obstante, el resultado obtenido fue contrario al esperado, ya que los filamentos bacteriales crecían mientras que se detenía la división celular. Posteriores trabajos de Rosenberg, mostraron que la formación de los complejos cis-diclorodiaminoplatino(II) y cis-tetraclorodiaminoplatino(IV) eran los responsables de inducir el crecimiento de los filamentos bacteriales en ausencia del campo eléctrico. Para la época, era un hecho experimental conocido que los compuestos químicos que producían filamentación en bacterias exhibían actividad anticancerígena (Rosenberg et al., 1969). Los experimentos motivaron la aplicación de estos compuestos en cobayos donde previamente se habían implantado sarcomas sólidos obteniéndose resultados satisfactorios (Rosenberg et al., 1969). Consecuentemente, decidió probarse en humanos con lo cual se dio paso a un nuevo campo de la medicina, el cual envuelve a la bioinorgánica y la quimioterapia del cáncer. Así, el fármaco llamado platinol o cisplatino, recibió la aprobación en 1979 por la Agencia de Alimentos y Medicamentos (FDA, por sus siglas en inglés) consolidándose hasta la fecha como un paradigma en el tratamiento de carcinomas testiculares (Higby et al., 1974; Luo et al., 2012; Fantini et al., 2011). Sin embargo, aunque su efectividad de curación es superior al $90 \%$ para tumores sólidos, presenta algunas desventajas que limitan su aplicación terapéutica; entre las cuales se reportan la resistencia innata o adquirida por la célula tumoral frente a la acción del fármaco y algunos efectos secundarios tales como: nefrotoxicidad, neurotoxicidad, ototoxicidad y naúseas (Videhult et al., 2006; Knight et al., 2005). Por ello, con el fin de expandir la efectividad de cisplatino y disminuir sus efectos secundarios, se han sintetizado alrededor de 3000 compuestos derivados con propiedades fisicoquímicas mejoradas en relación a cisplatino (Liu et al., 2008; Reed et al., 1986). Estos derivados presentan algunas modificaciones estructurales tanto de los ligandos portadores (aminos) como de los ligandos salientes (cloruros). Así por ejemplo, en carboplatino considerado como la segunda generación de fármacos anticancerosos basados en platino, los ligandos cloruro son reemplazados por el grupo ciclobutanodicarboxilato, el cual se cree que es el responsable de la disminución sustancial de los efectos nefrotóxicos de este fármaco en relación a cisplatino, pero sin que disminuya su actividad antitumoral (Sarmah y Deka, 2008). El mecanismo de acción de cisplatino y derivados con actividad anticancerosa ha sido objeto de extensiva investigación teórica y experimental, pues sus bases moleculares aún no ha sido completamente elucidadas (Lopes et al., 2008; Cerón-Carrasco et al., 2012; Mansouri-Torshizi et al., 2011; Kozelka et al., 1999; Barnes y Lippard 2004; Park y Lippard 2012; Gómez-Ruiz et al., 2012; Tanida et al., 2012; Jungwirth et al., 2012; Hou et al., 2012). Tradicionalmente, se ha aceptado que el mecanismo de acción de cisplatino transcurre en dos etapas; una etapa inicial que corresponde a la hidrólisis, considerada la etapa de activación, y una segunda etapa que involucra su interacción con el ácido desoxirribonucleico (ADN), induciendo importantes cambios sobre la estructura helicoide de este, lo que trae como consecuencia que se inhiban los procesos celulares de replicación y transcripción, conduciendo finalmente la célula tumoral a un proceso de apoptosis o muerte celular programada. El sitio de interacción para la unión de cisplatino al ADN corresponde a dos bases guaninas adyacentes en la posición N7 del anillo imidazolio (Gumus et al., 2009; Gómez-Ruiz et al., 2012). La elucidación de estos aspectos mecanísticos constituye una herramienta clave para el diseño racional de nuevos medicamentos basados en platino y que básicamente están supeditadas al conjunto de reglas empíricas de estructura-actividad establecidas por Cleare y Hoeschele (Monti et al., 2005). Recientemente, se han reportado nuevas estrategias para la síntesis de estos complejos anticancerosos, donde una de las aproximaciones particularmente interesantes, ha sido la coordinación de ligandos bioactivos sobre el centro de platino (Margiotta et al., 2010). Entre este tipo de ligantes, los aminoácidos han presentado resultados satisfactorios en cuanto a actividad y especificidad (van Zutphen y Reedijk, 2005; Galanski y Keppler, 2007). Los aminoácidos y sus derivados son excelentes candidatos en protocolos de síntesis para derivados de cisplatino bajo este esquema de coordinación, debido en parte a la marcada afinidad de quelatación que presentan estas moléculas hacia el platino(II) (Dalla Via et al., 2006). Además, este grupo de complejos han mostrado que mejoran su solubilidad en agua. Por lo tanto, es claro que una comprensión adecuada de la naturaleza reactiva de estas moléculas es fundamental para diseñar nuevas rutas sintéticas y comprender los mecanismos de acción de este tipo de fármacos. Sin embargo a pesar de la importancia de estos compuestos, pocos trabajos se han reportado al respecto a nivel experimental y teórico (Moradell et al., 2004; Pucci et al., 2008; Dalla Via et al., 2006). En este sentido, el presente trabajo se orienta hacia el estudio computacional de la reactividad química, de un grupo de complejos derivados de cisplatino con ligandos aminoácidos cuya actividad citostática ha sido establecida experimentalmente, usando la teoría del funcional de la densidad de la reactividad química (Broeckaert et al., 2008; Moens et al., 2007). Con este estudio, se espera avanzar en la compresión de las vías de reacción seguidas por estas especies y propiedades de unión al ADN. En razón a lo anterior se calculan el conjunto de propiedades globales de la 
reactividad para los complejos clínicamente usados cisplatino y carboplatino, como fármacos de referencias, incluyendo algunos complejos derivados donde se ha variado la naturaleza del ligando portador y manteniendo los ligandos cloruro como grupos salientes.

\section{METODOLOGÍA}

\section{Fundamentos Teóricos}

La teoría del funcional de la densidad, (DFT, por sus siglas en inglés), ha llegado a ser una herramienta indispensable para llevar a cabo cálculos de estructura electrónica de átomos y moléculas (Gázquez, 2008). Asimismo, ha proporcionado fundamentos sólidos para caracterizar la reactividad química dado que sin ambigüedad ni escalas arbitrarias, se han podido definir conceptos básicos de reactividad tales como electronegatividad y dureza (Parr y Weitao, 1994). Así, la reactividad química inherente de una determinada especie se puede expresar en términos de las derivadas de la energía total con respecto al número de electrones a potencial externo constante. Se observa entonces que la DFT proporciona un marco adecuado para el cálculo de estas derivadas (Parr y Weitao, 1994). De esta manera y basándose en el primer teorema de Hohenberg y Kohn (Hohenberg y Kohn, 1964), el cual permite escribir la energía de un sistema de electrones sujetos a la influencia de un potencial externo $v(r)$ en la forma:

$$
E[\rho]=F[\rho]+\int d r \rho(r) v(r)
$$

donde F[p], es el funcional universal de Hohenberg y Kohn (Gázquez, 2008) que viene dado en términos de la suma del funcional de la energía cinética y el funcional de la energía de interacción electrón-electrón. Por otro lado basándose en el segundo teorema de Hohenberg y Kohn (Zhou, 2012) se busca la energía y densidad del estado basal, de tal manera que se debe buscar la densidad que minimice la ecuación 1 , manteniendo el potencial externo fijo. Si la búsqueda se efectúa con restricciones, estas pueden introducirse mediante la técnica de los multiplicadores de Lagrange (Ito y Kunisch, 2008). Si la minimización ocurre sujeta a las condiciones de un número fijo de electrones de tal manera que se garantice la normalización de la densidad electrónica, es decir, $\mathrm{N}=\int \mathrm{dr} \rho(r)$ la energía del estado basal puede obtenerme a partir de la solución de la ecuación Euler-Lagrange quedando de la forma:

$\mu=\left(\frac{\delta E}{\delta \rho(r)}\right)_{v}=v(r)+\frac{\delta F}{\delta \rho(r)}$

donde $\mu$, el multiplicador indeterminado de Lagrange, es el potencial químico electrónico que mide la tendencia de escape de electrones del sistema desde una región con alto potencial químico a una con bajo potencial químico hasta el punto en el cual $\mu$ llega a ser constante en cualquier parte del espacio. El potencial químico también se puede definir como:

$\mu=\left(\frac{\partial \mathrm{E}}{\partial \mathrm{N}}\right)_{\mathrm{v}}$

Esta última definición es importante porque la curva experimental de la energía $(E)$ contra el número de electrones $(\mathrm{N})$ presentada en la figura 1, solo puede dibujarse para valores enteros de N (Solà, 2001). Si se conectan estos diferentes puntos mediante una curva continua, entonces el potencial químico electrónico es la pendiente de la curva en el punto $\mathrm{N}$.

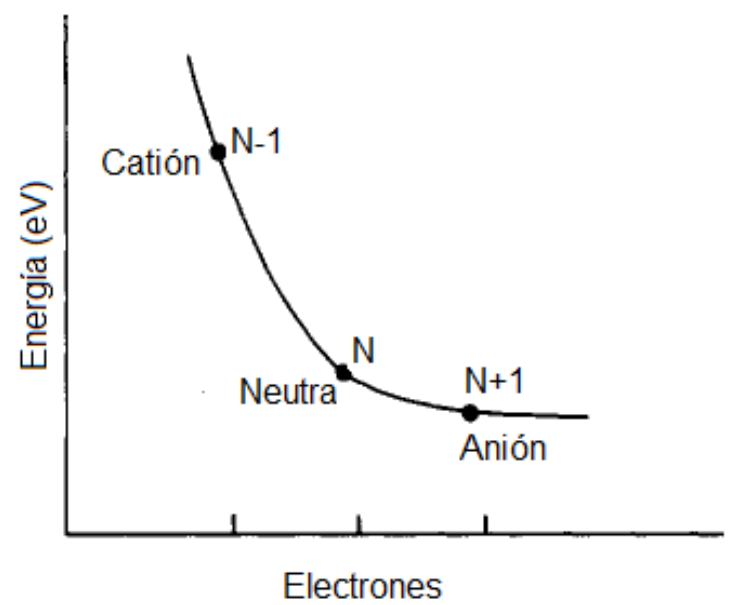

Fig. 1: Energía vs. número total de electrones 
Las diferencias de energía $E_{(N)}-E_{(N-1)}$ y $E_{(N)}-E_{(N+1)}$, corresponden al potencial ionización y la afinidad electrónica designados como I y A respectivamente. Estas cantidades se usan para calcular de forma aproximada la derivada parcial de la energía con respecto al número de electrones aplicando el método de diferencias finitas (Thomas, 1995). De esta aproximación, se obtiene la siguiente expresión:

$\mu=-\frac{(A+I)}{2}=-X_{M}$

De la ecuación 4, es evidente que el potencial químico electrónico por diferencias finitas equivale a la electronegatividad definitiva por Mulliken con signo menos (Mendoza-Huizar y Rios-Reyes, 2011). Ahora bien, como se indicó previamente, la DFT permite llegar al concepto de dureza química (n) (Parr y Weitao, 1994). Esto se logra con la segunda derivada de la energía con respecto al número de electrones a potencial externo constante que es:

$\eta=\frac{1}{2}\left(\frac{\partial^{2} E}{\partial N^{2}}\right)_{v}=\frac{1}{2}\left(\frac{\partial \mu}{\partial N}\right)_{v}$

De la ecuación 5, la dureza se puede interpretar como la resistencia del potencial químico electrónico de un sistema a cambiar con respecto al cambio en el número de electrones. Análogamente como se desarrolló para el potencial químico electrónico, el cálculo aproximado de esta derivada parcial por diferencias finitas conduce a la siguiente expresión:

$\eta=\frac{I-A}{2}$

Se puede afirmar entonces a partir de las ecuaciones 4 y 6 que el potencial químico electrónico mide la tendencia de una molécula a ceder o aceptar electrones provenientes de los alrededores, mientras que la dureza es una medida de la resistencia de la especie química a modificar su configuración electrónica. La validez de estos conceptos derivados de la DFT, se hace notoria cuando la información experimental disponible de I y A, se emplea para calcular el potencial químico electrónico y la dureza. Así por ejemplo, se observa que las cantidades obtenidas por la ecuación 4, siguen una tendencia similar a las electronegatividades calculadas por Pauling y los valores obtenidos de la dureza usando la expresión 6 , siguen la misma tendencia al concepto de dureza enunciado por Pearson (Pearson, 1997). También, es factible usar expresiones para el potencial químico electrónico y la dureza en términos de energías orbitales, mediante el teorema de Koopmans (Pain, 2011), donde los términos I y A, se toman como el negativo de las energías de los orbitales frontera HOMO y LUMO respectivamente. Bajo este esquema, se obtienen las expresiones 7 y 8 como se encuentran definidas en las referencias (Vivas-Reyes, 2008; Sarmah y Deka, 2008).

$\mu=\frac{E_{\text {LUMO }}+E_{\text {HOMO }}}{2}$
$\eta=\frac{E_{\text {LUMO }}-E_{\text {HOMO }}}{2}$

De las ecuaciones 7 y 8 , el término $E_{L U M O}$, es la energía del orbital desocupado de más baja energía y Е номо corresponde a la energía del orbital molecular ocupado de más alta energía respectivamente. Por otro lado, ya que el mecanismo de unión del fármaco cisplatino y derivados sobre ADN implica el ataque nucleofílico al centro de platino, se usó el índice de electrofilicidad global $(\omega)$ definido por Parr y colaboradores (Parr y Weitao, 1994). Este descriptor, a diferencia de $\mu$ y $\eta$ donde se mide la capacidad de la especie química para aceptar o donar un electrón, el índice de electrofilicidad mide el cambio en la energía de un electrófilo cuando este se satura con electrones. Parr, consideró que la especie química se encontraba inmersa en un baño de electrones con $\mu=0$; de tal modo que la especie recibía electrones hasta el punto en el cual el potencial químico electrónico llegaba a ser igual al potencial químico del baño. En este contexto, el índice de electrofilicidad puede ser expresado mediante la ecuación 9, como se encuentra definido en las referencias (Chattaraj y Giri, 2009; Chattaraj et al., 2006).

$\omega=\frac{\mu^{2}}{2 \eta}$

\section{Detalles Computacionales}

Los cálculos computacionales se llevaran a cabo con el paquete mecanocuántico Gaussian 03 (rev. E.01) (Frisch, 2004), optimizando sin restricciones a la geometría los complejos estudiados mediante el funcional 
de la densidad híbrido de un parámetro mPW1PW, introducido por Adamo y Barone (Padrão et al., 2011). Las estructuras optimizadas se caracterizaron como mínimos mediante cálculos de frecuencias vibracionales armónicas. Los descriptores de la reactividad globales, tales como el potencial químico, dureza e índice de electrofilicidad, se calcularon mediante las ecuaciones 7,8 y 9 respectivamente. A partir de las geometrías optimizadas en fase gas se reoptimizaron las geometrías de los complejos en fase acuosa usando el modelo de solvente del continuo polarizado PCM (por sus siglas en ingles) (Tomasi et al., 2005) al mismo nivel de teoría, y con una constante dieléctrica de 78.5 a una temperatura de $25^{\circ} \mathrm{C}$ correspondiente a la constante dieléctrica experimental del agua a dicha temperatura. Asimismo, se usó el potencial efectivo del core LANL2DZ de Hay y Wadt (Hay y Wadt, 1985) para reemplazar los 60 electrones de la capa más interna del átomo de platino y para los 18 electrones de valencia restantes se incluyeron implícitamente en el cálculo usando la base doble $\zeta$, asociada a LANL2DZ. A su vez, la base LANL2DZ se aumentó por una función de polarización d con un exponente a de 0.0747 para platino (Wysokiński y Michalska, 2001). Para el resto de átomos como lo son: cloro, carbono, nitrógeno, oxígeno e hidrógeno se usó la base doble $\zeta$ polarizada 6-31G(d).

\section{RESULTADOS Y DISCUSIÓN}

Los complejos estructuras de los estudiados se muestran en la figura 2. Para mayor claridad en la descripción de los parámetros geométricos y posterior comparación con la información experimental disponible de difracción de rayos X, los complejos (1), (2) y (14) se numeraron. Asimismo, a cada complejo le fue asignado un número en paréntesis con el propósito de ofrecer claridad en el análisis de los resultados.<smiles>N[PH3](N)(Cl)Cl</smiles>

(1)<smiles>[H][Y]1(Cl)NCC(C(=O)OCC)[R]1Cl</smiles>

(4)<smiles>[2H][Pb]1(N)OC(=O)C2(CCC2)C(=O)O1</smiles>

(2)<smiles>[H][Y]1(Cl)CC(C(=O)O)N[Y]1([H])Cl</smiles>

(3)<smiles>O=C(O)C1CCN[P+](Cl)(Cl)N1</smiles>

(5)<smiles></smiles>

(6)<smiles>CC(=O)OC(C)NC(=O)C1C[NH2+][PH](Cl)(Cl)N1</smiles>

(7)<smiles>COC(=O)C1CC[NH2+][PH](Cl)N1</smiles>

(8)<smiles>CC(=O)OC(Cc1ccccc1)NC(=O)C1C[NH2+][P@H](Cl)N1</smiles>

(9)<smiles>COC(NC(=O)C1CCNN[PH2+]N1)c1ccccc1</smiles>

(10)<smiles>CC(NC(=O)C1C[NH+][PH](Cl)(Cl)N1)OC=O</smiles>

(11)<smiles>CC(NC(=O)C1CCNC2NN1[PH]2Cl)OC=O</smiles>

(12)<smiles>NCCC[C@@H]1N[P+](Cl)(Cl)OC1=O</smiles>

(13)<smiles>[2H][C@@]1(CCCN)C(=O)O[PH](Cl)(Cl)[Y]1(Cl)Cl</smiles>

(14)

Fig. 2: Complejos de cis-platino(II) estudiados

De la figura 2, los complejos cisplatino (1) y carboplatino (2), se tomaron como fármacos de referencia, mientras que los complejos (3) a (14) corresponden a la serie de derivados cuya actividad citotóxica fue previamente ensayada (Moradell et al., 2004; Dalla Via et al., 2006). En los compuestos de coordinación (3) a (6) se observa la variación de la longitud de la cadena sobre el ligando etilendiamino, así como, la naturaleza del sustituyente sobre dicho ligando (para el caso los ligandos correspondieron a dap = ácido 
diaminopropiónico o dab = ácido 2,3-diaminobutírico, respectivamente). De esta manera, los complejos (3), (4), (5) y (6) se designaron como; $\mathrm{PtCl}_{2}$ (dap), $\mathrm{PtCl}_{2}$ (Etdap), $\mathrm{PtCl}_{2}$ (dab) y $\mathrm{PtCl}_{2}$ (Etdab) respectivamente (Moradell et al., 2003). Los complejos (7) a (12) tienen coordinados los ligandos dap y dab, con la diferencia que sobre dichos ligandos se han unido los aminoácidos alanina y fenilalanina. De ahí que, se designen como $\mathrm{PtCl}_{2}$ (dap-Metala), $\mathrm{PtCl}_{2}$ (dab-Metala), $\mathrm{PtCl}_{2}$ (dap-Phe), $\mathrm{PtCl}_{2}$ (dab-Phe), $\mathrm{PtCl}_{2}$ (dap-ala) y $\mathrm{PtCl}_{2}$ (dab-ala) respectivamente. Los complejos (13) y (14), recientemente sintetizados por Dalla Via y colaboradores (Dalla Via et al. 2006), fueron designados como $\mathrm{PtCl}_{2}(\mathrm{~L}-\mathrm{N}, \mathrm{O})$-Ornitina y $\mathrm{PtCl}_{2}(\mathrm{D}-\mathrm{N}, \mathrm{O})$-Ornitina respectivamente.

En la tabla 1, se presentan los parámetros geométricos calculados en fase gas y acuosa para cisplatino, carboplatino y $\mathrm{PtCl}_{2}(\mathrm{~L}-\mathrm{N}, \mathrm{O})$-Ornitina. Además, se presentan los datos experimentales de las geometrías elucidadas por difracción de rayos $X$ reportados en literatura (Dalla Via et al., 2006; Sarmah y Deka, 2008) para los complejos que aparecen numerados en la figura 2. De la tabla 1, se observa que el nivel de teoría usado permitió reproducir los parámetros geométricos experimentales en muy buen acuerdo, ya que se obtuvieron geometrías cuadrado planares con ángulos próximos a los valores ideales de $90^{\circ}$ y $180^{\circ}$, así como distancias de enlace muy próximas a los valores experimentales. Además, es de nuestro conocimiento que este funcional ya había sido usado previamente por Wysokinski y Michalska (Wysokinski y Michalska, 2001) quienes indicaron que el protocolo mPW1PW/LANL2DZ, es claramente superior a otros funcionales (incluyendo el popular B3LYP), para determinar las geometrías de cisplatino y carboplatino respectivamente. En términos generales, se puede afirmar que la inclusión del solvente permitió (Song y Hu, 2006), mejorar la descripción de los parámetros geométricos, dado que las distancias de enlace Pt-Cl y Pt$\mathrm{N}$ calculadas para cisplatino, estuvieron en concordancia con los valores experimentales. Asimismo, para los ángulos de enlace calculados en fase acuosa para cisplatino, $\angle(\mathrm{Cl}-\mathrm{Pt}-\mathrm{Cl})$ y $\angle(\mathrm{N}-\mathrm{Pt}-\mathrm{N})$ mostraron desviaciones del orden de $1.37^{\circ}$ y $3.59^{\circ}$ con respecto al valor experimental. De igual manera, los parámetros geométricos predichos para carboplatino fueron próximos a los reportados en literatura (Sarmah y Deka, 2008).

Tabla 1: Parámetros geométricos calculados en fase gas y acuosa para los complejos 1, 2 y 14

\begin{tabular}{|c|c|c|c|}
\hline & \multicolumn{2}{|c|}{ Calculado con mPW1PW/LANL2DZ } & Experimental \\
\hline Parámetro geométrico & \multicolumn{2}{|c|}{ cisplatino } & (Sarma y Deka, 2008) \\
\hline Distancia de enlace & Fase Gas & Fase acuosa & --- \\
\hline $\mathrm{r}(\mathrm{Pt}-\mathrm{Cl})$ & 2.322 & 2.358 & $2.33 \pm 0.01$ \\
\hline$r(\mathrm{Pt}-\mathrm{N})$ & 2.089 & 2.055 & $2.01 \pm 0.04$ \\
\hline \multicolumn{4}{|l|}{ Ángulos de enlace } \\
\hline$\angle(\mathrm{Cl}-\mathrm{Pt}-\mathrm{Cl})$ & 95.182 & 93.270 & $91.9 \pm 0.3$ \\
\hline$\angle(\mathrm{N}-\mathrm{Pt}-\mathrm{N})$ & 98.931 & 92.096 & $87 \pm 1.5$ \\
\hline$\angle(\mathrm{N}-\mathrm{Pt}-\mathrm{Cl})$ & 177.727 & 179.588 & --- \\
\hline & \multicolumn{2}{|c|}{ carboplatino } & (Sarma y Deka, 2008) \\
\hline $\mathrm{r}(\mathrm{Pt}-\mathrm{N} 1)$ & 2.086 & 2.055 & 2.01 \\
\hline r(Pt-O1) & 2.087 & 2.012 & 2.02 \\
\hline$\angle(\mathrm{O} 1-\mathrm{Pt}-\mathrm{N} 1)$ & 80.026 & 87.512 & 88.20 \\
\hline$\angle(\mathrm{N} 1-\mathrm{Pt}-\mathrm{N} 2)$ & 103.684 & 92.914 & 93.60 \\
\hline$\angle(\mathrm{O} 1-\mathrm{Pt}-\mathrm{N} 2)$ & 175.456 & 177.946 & 177.90 \\
\hline \multirow[t]{2}{*}{$\angle(\mathrm{O} 1-\mathrm{Pt}-\mathrm{O} 2)$} & 96.267 & 92.046 & 88.90 \\
\hline & \multicolumn{2}{|c|}{$\mathrm{PtCl}_{2}(\mathrm{~L}-\mathrm{N}, \mathrm{O})-$ Ornitina } & (Dalla Via et al., 2006) \\
\hline $\mathrm{r}(\mathrm{Pt}-\mathrm{Cl} 1)$ & 2.303 & 2.345 & 2.317 \\
\hline $\mathrm{r}(\mathrm{Pt}-\mathrm{Cl} 2)$ & 2.276 & 2.298 & 2.292 \\
\hline r(O1-C1) & 1.376 & 1.380 & 1.280 \\
\hline r(C1-O2) & 1.194 & 1.193 & 1.260 \\
\hline r(Pt-01) & 2.154 & 2.105 & 2.036 \\
\hline r(Pt-N1) & 2.084 & 2.055 & 2.060 \\
\hline$r(\mathrm{~N} 1-\mathrm{C} 2)$ & 1.483 & 1.486 & 1.480 \\
\hline$r(C 1-C 2)$ & 1.523 & 1.516 & 1.530 \\
\hline$\angle(\mathrm{Cl} 1-\mathrm{Pt}-\mathrm{Cl} 2)$ & 95.700 & 92.518 & 92.4 \\
\hline$\angle(\mathrm{O} 1-\mathrm{Pt}-\mathrm{N} 1)$ & 79.719 & 79.197 & 83.1 \\
\hline$\angle(\mathrm{Pt}-\mathrm{O} 1-\mathrm{C} 1)$ & 113.574 & 116.731 & 114.0 \\
\hline$\angle(\mathrm{Pt}-\mathrm{N} 1-\mathrm{C} 2)$ & 111.066 & 111.818 & 109.4 \\
\hline$\angle(\mathrm{Cl} 1-\mathrm{Pt}-\mathrm{N} 1)$ & 172.998 & 174.407 & 173.7 \\
\hline$\angle(\mathrm{Cl} 2-\mathrm{Pt}-\mathrm{O} 1)$ & 170.839 & 172.168 & 175.5 \\
\hline$\angle(\mathrm{O} 1-\mathrm{C} 1-\mathrm{C} 2)$ & 110.957 & 111.295 & 120.0 \\
\hline$\angle(\mathrm{N} 1-\mathrm{C} 2-\mathrm{C} 1)$ & 110.745 & 110.689 & 110.0 \\
\hline
\end{tabular}

Con respecto al complejo (14), cuyos parámetros geométricos predichos se reportan por primera vez, las distancias y ángulos de enlace fueron consistentes con la información experimental (Dalla Via et al., 2006). 
Nuevamente se hizo evidente, que en fase acuosa los resultados arrojados por el cálculo computacional mejoran en relación a los cálculos efectuados en fase gas. Además, cabe resaltar que el núcleo de platino en el complejo (14), está rodeado por un entorno cuadrado planar, así como lo confirman los ángulos próximos a $90^{\circ}$ y $180^{\circ}$ respectivamente. Los parámetros geométricos calculados para los complejos (2) a (13), y de los cuales no se dispone de datos de difracción de rayos X, sus geometrías se compararon con los complejos análogos de estructura química similar mostrando buen acuerdo.

En la tabla 2, se presentan los valores de potencial químico, dureza e índice de electrofilicidad calculados al nivel de teoría mPW1PW/LANL2DZ. Los valores de dureza para todos los complejos en la fase acuosa, excepto para carboplatino, indican que estas especies incrementan su estabilización en dicho medio. El complejo carboplatino fue la estructura más estable tanto en la fase gas como la acuosa, presentando el mayor valor de dureza de los complejos estudiados. Este resultado es consecuente con el principio de máxima dureza de Pearson (Pearson, 1997), según el cual la especie química más estable tiende al máximo de dureza. Asimismo, este complejo presenta el valor más bajo de $\omega$ y máximo de $\mu$. En la tabla 2 , se observa que cisplatino es más reactivo que carboplatino, teniendo en cuenta que los valores del índice de electrofilicidad y potencial químico electrónico son alrededor de 1.0 y 0.6 eV más altos que en carboplatino.

En este punto, es importante anotar que los datos de actividad citotóxica reportados por Moradell (Moradell et al., 2004) y Dalla (Dalla Via et al., 2006), indican que cisplatino es la especie con mayor actividad y por ende la más reactiva. Sin embargo, los descriptores de la reactividad revelan que los complejos (3), (5), (9), (13) y (14), muestran un comportamiento contrario al esperado, siendo más reactivos que el complejo patrón. Las diferencias más significativas se observan para los complejos (13) y (14) cuyos índices de electrofilicidad son aproximadamente $1.5 \mathrm{eV}$ mayores que los predichos para cisplatino. Obviamente, la naturaleza de los ligandos coordinados al $\mathrm{Pt}$, afectan su reactividad. De lo anterior es claro, que los aminoácidos, efectivamente incrementan la reactividad de los complejos como fue inicialmente sugerido en el trabajo de Moradell (Moradell et al., 2004). Sin embargo, el hecho que estos derivados incrementan su reactividad en relación a cisplatino no implica que su acción citostática mejore, ya que hay que considerar una serie de factores fisicoquímicos que pueden disminuir la actividad. Una explicación razonable para este resultado puede inferirse a partir del trabajo reportado por Moradell (Moradell et al., 2004), donde los complejos (9) y (10), presentaron una actividad citotóxica significativamente baja. Moradell sugirió, que esta disminución en la actividad de dicho complejo se debía a la cadena lateral voluminosa de fenilalanina, la cual dificultaba su paso través de la membrana celular. Entonces, se puede afirmar que la reactividad de los complejos derivados de cisplatino donde se coordina un ligando aminoácido aumenta su reactividad (tabla 2), pero en condiciones fisiológicas se debe tener en cuenta que una cadena lateral voluminosa no presenta un efecto positivo sobre la citotoxicidad del potencial fármaco (Moradell et al., 2004).

Tabla 2: Descriptores globales predichos para los complejos estudiados. Las unidades están expresadas en electrón-voltios $(\mathrm{eV})$

\begin{tabular}{cccc|ccc}
\hline \multirow{2}{*}{ Complejo } & \multicolumn{3}{c|}{ Fase Gas } & \multicolumn{3}{c}{ Fase Acuosa } \\
\cline { 2 - 7 } & $\mu(\mathrm{eV})$ & $\eta(\mathrm{eV})$ & $\omega(\mathrm{eV})$ & $\mu(\mathrm{eV})$ & $\eta(\mathrm{eV})$ & $\omega(\mathrm{eV})$ \\
\hline 1 & -3.837 & 2.585 & 2.847 & -4.177 & 2.816 & 3.097 \\
2 & -3.469 & 2.844 & 2.117 & -3.606 & 3.197 & 2.033 \\
3 & -3.782 & 2.449 & 2.921 & -4.218 & 2.776 & 3.205 \\
4 & -3.674 & 2.640 & 2.556 & -4.177 & 2.844 & 3.068 \\
5 & -3.674 & 2.476 & 2.725 & -4.218 & 2.776 & 3.205 \\
6 & -3.619 & 2.640 & 2.481 & -4.163 & 2.830 & 3.062 \\
7 & -3.497 & 2.517 & 2.429 & -4.163 & 2.803 & 3.092 \\
8 & -3.483 & 2.640 & 2.298 & -4.150 & 2.844 & 3.028 \\
9 & -3.551 & 2.490 & 2.532 & -4.177 & 2.789 & 3.128 \\
10 & -3.524 & 2.626 & 2.364 & -4.150 & 2.844 & 3.028 \\
11 & -3.551 & 2.517 & 2.505 & -4.150 & 2.844 & 3.028 \\
12 & -3.510 & 2.640 & 2.334 & -4.150 & 2.844 & 3.028 \\
13 & -4.286 & 2.218 & 4.141 & -4.572 & 2.313 & 4.518 \\
14 & -4.367 & 2.191 & 4.354 & -4.544 & 2.286 & 4.517 \\
\hline
\end{tabular}

Por otro lado, de los índices de electrofilicidad para los complejos (9) a (14), se observa que si el aminoácido esta unido directamente al átomo de platino, su reactividad es mayor que cuando este se une a 
los ligandos dap o dab respectivamente. Evidentemente, hay un efecto electro-atrayente del ligando, dado que la densidad de carga sobre el centro metálico disminuye y por ende su carácter electrofílico. El alto valor de electrofilia presentado por estos complejos también podría servir como base para explicar su bajo efecto citotóxico comparado con cisplatino, dado que pueden presentarse interacciones tipo electrófilo/nucleófilo con otras biomoléculas antes de alcanzar el ADN. Esta idea se puede soportar considerando que los principales mecanismos de resistencia observados para cisplatino se deben principalmente a interacciones no específicas con macromoléculas que contienen azufre, entre las cuales se pueden destacar glutatión y metalotioneínas (Huska et al., 2009). Ahora bien, un punto interesante para analizar es que cisplatino presenta una selectividad muy baja debido a su alta reactividad. Se esperaría entonces que los complejos (13) y (14) presenten la misma tendencia, sin embargo la evidencia experimental sugiere que estos fármacos derivados presentan mayor selectividad que cisplatino, debido a que el aminoácido ornitina es uno de los precursores en la biosíntesis natural de poliaminas y policationes, los cuales son altamente requeridos por células tumorales (Seiler et al., 1996). En consecuencia, el transporte de estos complejos con ligandos ornitina al interior de la célula es más eficientemente (Seiler et al., 1996). Esto último, soportaría el hecho que los complejos (13) y (14) tengan mayor actividad en relación a los complejos (9) a (12), aun cuando su tamaño molecular sea muy cercano (Dalla Via et al., 2006).

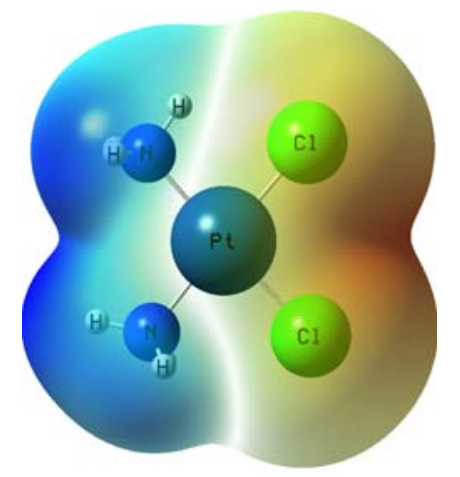

$2 a$.

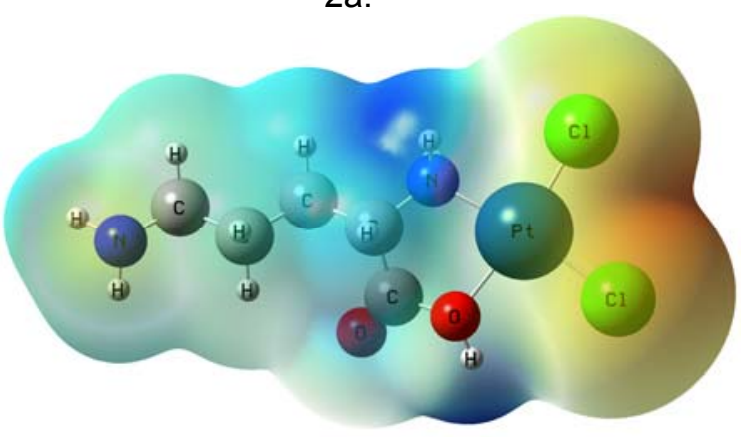

2c.

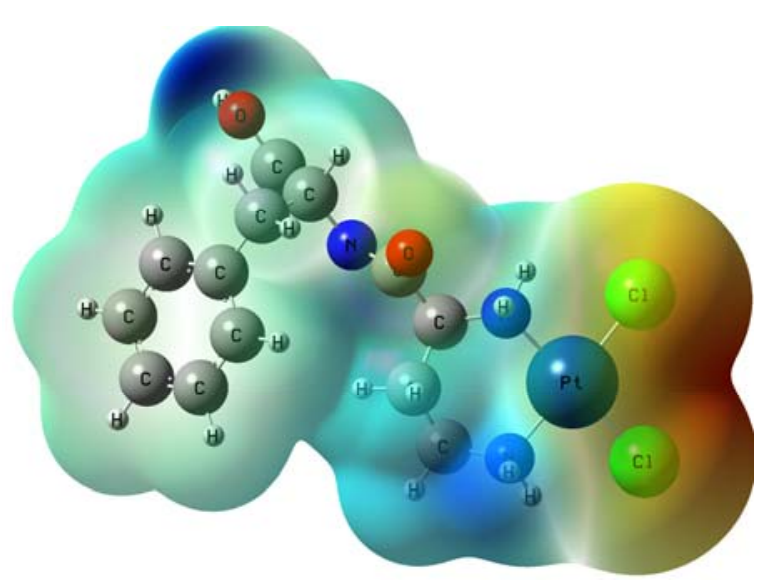

$2 b$.

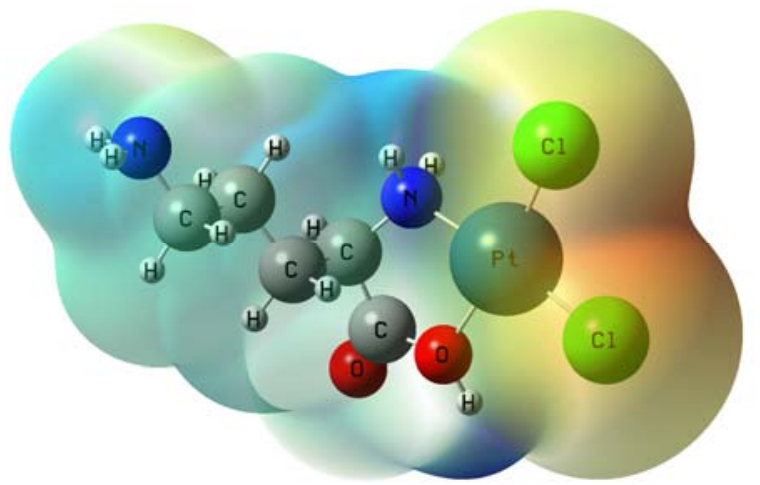

$2 d$

Fig. 3: Vista 3D de los mapas de potencial electrostático para el complejo cisplatin ${ }^{2 a}$ y los derivados $10^{(2 b)}$, $13^{(2 \mathrm{c})}$ y $14^{(2 \mathrm{~d})}$ respectivamente. (Valor de isosuperficie $=0.03 \mathrm{e} / \mathrm{ua}^{3}$ )

Para analizar la reactividad de estos complejos frente al ataque nucleofílico, se calcularon los mapas de potencial electrostático molecular (MEP, por sus siglas en inglés) (Vivas-Reyes y Zapata, 2008), al mismo nivel de teoría usado en el presente estudio. El potencial electrostático molecular es una herramienta básica para la interpretación de la reactividad molecular de especies electrofílicas, ya que, permite visualizar aquellas regiones de una molécula susceptibles a un ataque nucleofílico. En la figura 3 , se presentan los MEPs obtenidos para los complejos (1), (10), (13) y (14) respectivamente. El código de colores adoptado en la figura, muestra que las regiones con potenciales positivos, son aquellas en tonalidad azul y las regiones con potencial negativo las de color rojo. Como se esperaba, los átomos de platino son regiones con potencial positivo y por lo tanto susceptibles a ataques nucleofílicos, lo cual está en total acuerdo con lo predicho por los valores de electrofilicidad global, ya que, confirman el poder electrofílico de estos complejos y por lo tanto su propensión hacia el ataque nucleofílico por ADN. 
Otro aspecto importante que puede sugerirse a partir de la interpretación del MEP, es que el cambio en la naturaleza del ligando portador provee de más regiones positivas al complejo y por ende aumenta el número de sitios de interacción con ADN. Así por ejemplo, podría esperarse que los complejos (10), (13) y (14) induzcan una mayor distorsión sobre la hebra de ADN que cisplatino. En efecto, el resultado arrojado por el MEP para el complejo (10) se encuentra en línea con la evidencia experimental disponible sobre la interacción de este complejo con su diana biológica primaria; dado que los espectros de dicroísmo circular obtenidos por Moradell (Moradell et al., 2004) para dicho complejo, mostraron que induce fuertes distorsiones sobre la hebra de ADN en comparación con el fármaco cisplatino que solo induce pequeñas distorsiones. EI MEP predice este comportamiento, pues como se observa en la figura $2 \mathrm{~b}$ hay un mayor número de sitios donde la densidad electrónica es baja (regiones en azul) y por ende existe mayor probabilidad de un ataque nucleofílico.

\section{CONCLUSIONES}

Se puede concluir que el nivel de teoría mPW1PW/LANL2DZ, permitió determinar la reactividad, estabilidad y estructura geométrica de los complejos (1)-(14). Los parámetros geométricos predichos en fase acuosa para cisplatino, carboplatino y el derivado $\mathrm{PtCl}_{2}(\mathrm{~L}-\mathrm{N}, \mathrm{O}$-ornitina), fueron muy próximos a los experimentales en comparación con los calculados en fase gas. El comportamiento electrofílico de todos los complejos aumentó en la fase acuosa, excepto para carboplatino. Los complejos (13) y (14) presentaron valores de $\omega$ relativamente altos comparados con los de cisplatino y el resto de complejos. Una explicación plausible para este resultado, es que la unión de aminoácidos en la esfera de coordinación primaria del complejo induce un efecto favorable en su reactividad, debido a su carácter electro-atrayente. De ahí que, los complejos (9), (10), (11) y (12) donde los aminoácidos alanina y fenilalanina no están unidos directamente al complejo fueron menos reactivos.

\section{AGRADECIMIENTOS}

Los autores de este artículo muestran su agradecimiento al Centro de Investigaciones Universitarias (CIUC), de la Universidad de Córdoba, por el soporte financiero durante la ejecución de este trabajo (proyecto de investigación código 1.2.08.109, numeral FCB-03-09).

\section{REFERENCIAS}

Abramkin, S.A, U. Jungwirth, U. Valiahdi y otros diez autores, (1R,2R,4R)-4-Methyl-1,2-cyclohexanediamine) oxalatoplatinum(II): A Novel Enantiomerically Pure Oxaliplatin Derivative Showing Improved Anticancer Activity in Vivo, J. Med. Chem: 53(20), 7356-7364 (2010).

Beck, D.J. y R.R. Brubaker, Effect of cis-Platinum(II)Diamminodichloride on Wild Type and Deoxyribonucleic Acid Repair-Deficient Mutants of Escherichia coli1, J. Bacteriol: 116(3), 1247-1252 (1973).

Broeckaert, L., J. Moens, G. Roos, F. De Proft y P. Geerlings, Intrinsic nucleofugality scale within the framework of density functional reactivity theory, J. Phys. Chem A: 112(47), 12164-12171 (2008).

Barnes, Katie R, y Stephen J Lippard, Cisplatin and related anticancer drugs: recent advances and insights, Met. Ions. Biol. Syst: 42, 143-177 (2004).

Cerón-Carrasco, J.P., D. Jacquemin y E. Cauët, Cisplatin cytotoxicity: a theoretical study of induced mutations, Phys. Chem. Chem. Phys: 14(36), 2457-12464 (2012).

Chattaraj, P.K. y S. Giri, Electrophilicity index within a conceptual DFT framework, Annual Reports Section C Physical Chemistry: 105(0), 13-39 (2009).

Chattaraj, P.K., U. Sarkar y D.R. Roy, Electrophilicity Index, Chem. Rev: 106(6), 2065-2091 (2006).

Dalla Via, L., O. Gia, S.M. Magno, A. Dolmella y M.V. Di Noto, Synthesis, characterization and biological activity of platinum(II) complexes with I- and d-ornithine ligands, Inorg. Chim. Acta: 359(13), 4197-4206 (2006).

Fantini, M., L. Gianni y otros seis autores, Lipoplatin Treatment in Lung and Breast Cancer, Chemother Res Pract: 1-7 (2011).

Frisch, M. J., Gaussian 03, Rev. E.01, Wallingford CT: Gaussian, Inc (2004). 
Galanski, M. y B.K. Keppler, Searching for the magic bullet: anticancer platinum drugs which can be accumulated or activated in the tumor tissue, Anticancer Agents Med Chem: 7(1), 55-73 (2007).

Gázquez, J.L., Perspectives on the Density Functional Theory of Chemical Reactivity, J. Mex. Chem. Soc: 52(1), 3-10 (2008).

Gómez-Ruiz, S., D. Maksimović-Ivanić, S. Mijatović, y G.N. Kaluđerović, On the Discovery, Biological Effects, and Use of Cisplatin and Metallocenes in Anticancer Chemotherapy, Bioinorg Chem Appl: 1-14 (2012).

Gumus, F, G. Eren y otros nueve autores, Synthesis, Cytotoxicity, and DNA Interactions of New Cisplatin Analogues Containing Substituted Benzimidazole Ligands, J. Med. Chem: 52(5), 1345-1357 (2009).

Hay, P.J. y W.R. Wadt, Ab initio effective core potentials for molecular calculations. Potentials for the transition metal atoms Sc to Hg, J. Chem. Phys: 82(1), 270-283 (1985).

Higby, D. J., H.J. Wallace, D.J. Albert y J.F. Holland, Diaminodichloroplatinum: A phase I study showing responses in testicular and other tumors, Cancer: 33(5), 1219-1225 (1974).

Hohenberg, P. y W. Kohn, Inhomogeneous Electron Gas, Phys. Rev: 136(3B), B864-B871 (1964).

Hou, X.M. X. Zhang y otros seis autores, Cisplatin induces loop structures and condensation of single DNA molecules, Nucleic. Acids. Res: 37(5), 1400-1410 (2009).

Huska, D., I. Fabrik y otros nueve autores, Study of Interactions between Metallothionein and Cisplatin by using Differential Pulse Voltammetry Brdicka's reaction and Quartz Crystal Microbalance, Sensors: 9(3), 1355-1369 (2009).

Hay, P. y W.R. Wadt, Ab initio effective core potentials for molecular calculations. Potentials for the transition metal atoms Sc to Hg, J. Chem. Phys: 82(1), 270-283 (1985).

Ito, K. y K. Kunisch, Lagrange multiplier approach to variational problems and applications, Society for Industrial and Applied Mathematics (2008).

Jungwirth, U., D. Xanthos y otros once autores, Anticancer Activity of Methyl-Substituted Oxaliplatin Analogs, Mol. Pharmacol: 81(5), 719-728 (2012).

Knight, K.R, D.F. Kraemer y E.A. Neuwelt, Ototoxicity in Children Receiving Platinum Chemotherapy: Underestimating a Commonly Occurring Toxicity That May Influence Academic and Social Development, J. Clin. Oncol: 23(34), 8588-8596 (2005).

Kozelka, J., F. Legendre, F. Reeder y J.C. Chottard, Kinetic aspects of interactions between DNA and platinum complexes, Coord. Chem. Rev: 190-192(0), 61-82 (1999).

Liu, W.P., Q. Ye y otros ocho autores, Novel Lipophilic Platinum(II) Compounds of Salicylate Derivatives, Platinum. Metals. Review: 52(3), 163-171 (2008).

Lopes, J.F., W.R. Rocha, H.F. Dos Santos y W.B. De Almeida, Theoretical study of the potential energy surface for the interaction of cisplatin and their aquated species with water, J. Chem. Phys: 128(16), 165103-165103 (2008).

Luo, T., J. Yu y otros siete autores, Electron transfer-based combination therapy of cisplatin with tetramethylp-phenylenediamine for ovarian, cervical, and lung cancers, PNAS: 109(26), 10175-10180 (2012).

Mansouri-Torshizi, H., y otros cinco autores, DNA Binding and Antitumor Activity of $\alpha$-Diimineplatinum(II) and Palladium(II) Dithiocarbamate Complexes, Bioinorg. Chem. Appl: 1-11 (2011).

Margiotta, N., N. Denora y otros seis autores, Platinum(II) complexes with bioactive carrier ligands having high affinity for the translocator protein, J. Med. Chem: 53(14), 5144-5154 (2010).

Mendoza-Huizar, L.H. y C.H. Rios-Reyes, Chemical Reactivity of Atrazine Employing the Fukui Function, J. Mex. Chem. Soc: (3), 142-147 (2011). 
Moens, J., P. Geerlings y G. Roos, A conceptual DFT approach for the evaluation and interpretation of redox potentials, Chemistry: 13(29), 8174-8184 (2007).

Monti, E., M. Gariboldi y otros cinco autores, Cytotoxicity of cis-Platinum(II) Conjugate Models. The Effect of Chelating Arms and Leaving Groups on Cytotoxicity: A Quantitative Structure-Activity Relationship Approach, J. Med. Chem: 48(3) 857-866 (2005).

Moradell, S., J. Lorenzo y otros ocho autores, Platinum complexes of diaminocarboxylic acids and their ethyl ester derivatives: the effect of the chelate ring size on antitumor activity and interactions with GMP and DNA, J. Inorg. Biochem: 96(4), 493-502 (2003)

Moradell, S., J. Lorenzo y otros ocho autores, Water-soluble platinum(II) complexes of diamine chelating ligands bearing amino-acid type substituents: the effect of the linked amino acid and the diamine chelate ring size on antitumor activity, and interactions with 5'-GMP and DNA, J. Inorg. Biochem: 98(11), 1933-1946 (2004).

Padrão, S, S.M. Fiuza, A.M. Amado, A.M. Amorim da Costa y L. A. E. Batista de Carvalho, Validation of the MPW1PW Quantum chemical calculations for the vibrational study of organic molecules re-assignment of the isopropylamine vibrational spectra, J. Phys. Org. Chem: 24(2), 110-121 (2011).

Park, S, y S.J Lippard, Binding interaction of HMGB4 with cisplatin-modified DNA, Biochemistry: 51(34): 6728-6737 (2012).

Pain, J.C., Koopmans' theorem in statistical Hartree-Fock theory, arXiv:1106-1155 (2011).

Parr, R.G. y Y. Weitao, Density-Functional Theory of Atoms and Molecules, Oxford University Press, USA (1994).

Pearson, R.G., Chemical Hardness: Applications from Molecules to Solids, $1^{\text {a }}$ edición, Wiley-VCH (1997).

Pucci, D. A. Belluci y otros ocho autores, Bioactive fragments synergically involved in the design of new generation Pt(II) and Pd(II)-based anticancer compounds, Dalton Trans: (43), 5897-5904 (2008).

Reed, E, S.H. Yuspa, L.A. Zwelling, R.F. Ozols y M.C. Poirier, Quantitation of cis-diamminedichloroplatinum II (cisplatin)-DNA-intrastrand adducts in testicular and ovarian cancer patients receiving cisplatin chemotherapy, J. Clin. Invest: 77(2), 545-550 (1986).

Rosenberg, B., L. Vancamp, J.E. Trosko y V.H. Mansour, Platinum Compounds: a New Class of Potent Antitumour Agents, Nature: 222(5191), 385-386 (1969).

Seiler, N, J.G. Delcros y J.P. Moulinoux, Polyamine transport in mammalian cells. An update, Int. J. Biochem. Cell. Biol: (28), 843-861 (1996).

Solà, M., Principios fundamentales de reactividad química basados en la estructura electrónica de las moléculas, Anales RSEQ: (4), 12-18 (2001).

Sarmah, P y R.C. Deka, Solvent effect on the reactivity of cis-platinum (II) complexes: A density functional approach, Int. J. Quantum. Chem: 108(8), 1400 - 1409 (2008).

Song, T y P. Hu, Insight into the solvent effect: A density functional theory study of cisplatin hydrolysis, J.

Chem. Phys: 125(9), 091101 (2006).

Tanida, S., T. Mizoshita y otros seis autores, Mechanisms of Cisplatin-Induced Apoptosis and of Cisplatin Sensitivity: Potential of BIN1 to Act as a Potent Predictor of Cisplatin Sensitivity in Gastric Cancer Treatment, Int J Surg Oncol: 1-8 (2012).

Thomas, J.W., Numerical Partial Differential Equations: Finite Difference Methods, Springer (1995)

Tomasi, J, B. Mennucci y R. Cammi, Quantum Mechanical Continuum Solvation Models, Chem. Rev: 105(8) 2999-3094 (2005). 
Videhult, P., G. Laurell, I. Wallin y H. Ehrsson, Kinetics of Cisplatin and Its Monohydrated Complex with Sulfur-Containing Compounds Designed for Local Otoprotective Administration, Exp. Biol. Med: 231(10), 1638-1645 (2006).

Vivas-Reyes, R. y J. Zapata, Estudio teórico de la reactividad de las conformaciones y configuraciones de los ácidos grasos omega-3 a través de descriptores moleculares de reactividad utilizando la Teoría del Funcional de la Densidad (DFT), Rev. Colomb. Quim: 37(2), 145-160 (2008).

van Zutphen, S y J. Reedijk, Targeting platinum anti-tumour drugs: Overview of strategies employed to reduce systemic toxicity, Coord. Chem. Rev: 249(24), 2845-2853 (2005).

Wysokiński, R y D. Michalska, The performance of different density functional methods in the calculation of molecular structures and vibrational spectra of platinum(II) antitumor drugs: cisplatin and carboplatin, J. Comput. Chem: 22(9), 901-912 (2001).

Zhou, A., Hohenberg-Kohn theorem for coulomb type systems and its generalization, J. Math. Chem: 50(10), 2746-2754 (2012). 